\title{
A comparison of triangular and quadrilateral finite element meshes for Bragg edge neutron transmission strain tomography
}

\author{
$\begin{array}{ll}\text { R. Aggarwal } & \text { B. P. Lamichhane } \\ & \text { M. H. Meylan }\end{array}$ \\ C. M. Wensrich ${ }^{4}$
}

(Received 26 February 2020; revised 16 August 2020)

\begin{abstract}
A wavelength resolved measurement technique used in neutron imaging applications is known as energy-resolved neutron transmission imaging. This technique of reconstructing residual strain maps provides high spatial resolution measurements of strain distribution in polycrystalline materials from sets of Bragg edge measurement images. Strain field reconstructions obtained from both triangular and quadrilateral finite element meshes are compared. The reconstruction is approached via a least square method and relies on the inversion of the longitudinal ray transform, which has uniqueness issues.
\end{abstract}

DoI:10.21914/anziamj.v61i0.15171, (C) Austral. Mathematical Soc. 2020. Published 2020-08-30, as part of the Proceedings of the 14th Biennial Engineering Mathematics and Applications Conference. ISSN 1445-8810. (Print two pages per sheet of paper.) Copies of this article must not be made otherwise available on the internet; instead link directly to the DOI for this article. 


\section{Contents}

1 Introduction

C243

2 Longitudinal ray transform

C244

3 Finite element discretisation

C245

4 Simulation results for a test case

C249

\section{Introduction}

This research presents a technique for measuring spatially resolved structures in crystalline materials using pulsed neutron beams based on the pixelated time-of-flight (ТОF) neutron transmission method. The TOF is the total time taken by a neutron while travelling from the sample to the detector. Tomographic imaging deals with reconstructing an image from its projections. From a mathematical viewpoint, neutron strain tomography examines the data accumulated by a ray to best extract information about strain. This work is motivated by the need to reconstruct three-dimensional maps of bulk variation in residual elastic strain throughout samples like steel cylinders, ring-and-plugs, and crushed rings using two-dimensional projected data [10, $14,13]$.

On a pulsed source $[12,11]$, energy-resolved imaging is enabled by a TOF analysis of the neutrons transmitted or scattered by the sample. By measuring neutron TOF and calculating an associated wavelength from measured neutron velocity, the wavelength-intensity distribution at the detector can be determined. The relative transmission of a neutron is observed as a function of wavelength and sharp discontinuities in transmission indicate Bragg edges. These Bragg edges occur at points where Bragg's law can no longer be fulfilled. The Bragg edge position for a given wavelength is directed by diffraction and provides a direct measure of lattice parameters [7, 4]. Reconstruction of strain from energy-resolved imaging has its limitations, as it only measures 
the averaged strain through the sample thickness in the direction of neutron propagation [14].

The method proposed here, based on the Bragg edge neutron transmission method, is non-destructive and, in principle, allows three-dimensional reconstruction of the residual strain throughout the bulk of the sample. Although in the present work we consider reconstructing two-dimensional elastic strain using sets of Bragg images. The reconstruction involves the calculation of line integrals using a longitudinal ray transform (LRT). In the present work we compare the reconstructed strain field with different mesh types, namely triangular (three nodes) and quadrilateral (four nodes).

\section{Longitudinal ray transform}

The strain in the direction parallel to the neutron beam is determined by measuring the relative shifts in the position of Bragg edges in the TOF spectrum and relating them to shifts in the lattice spacing. The lattice spacing $d$ and the TOF $t$ share a linear relationship

$$
\mathrm{d}=\frac{\mathrm{h}}{2 \mathrm{~mL}} \mathrm{t}
$$

where $\mathrm{h}$ is Planck's constant, $\mathrm{m}$ is the neutron mass and $\mathrm{L}$ is the path length. The elastic strains are

$$
\epsilon=\frac{\mathrm{d}-\mathrm{d}_{0}}{\mathrm{~d}_{0}}=\frac{\triangle \mathrm{t}}{\mathrm{t}}
$$

where $d_{0}$ is the unstrained lattice spacing. Mathematically, the average strain within a body, as measured by Bragg edge neutron transmission, can be idealised as a line integral called the longitudinal ray transform (LRT), which captures the component of strain along the line in the direction of the unit vector $\hat{\mathbf{n}}=\left(\mathrm{n}_{1}, \mathrm{n}_{2}\right)$ [10]. The LRT of the strain field models each pixel within a strain image:

$$
I_{\epsilon}\left(x_{0}, y_{0}, \widehat{n}\right)=\frac{1}{\ell} \int_{0}^{\ell} \hat{n}^{\top} \epsilon\left(x_{0}+s n_{1}, y_{0}+s n_{2}\right) \hat{n} d s,
$$


where

$$
\epsilon(x, y)=\left[\begin{array}{ll}
\epsilon_{11}(x, y) & \epsilon_{12}(x, y) \\
\epsilon_{12}(x, y) & \epsilon_{22}(x, y)
\end{array}\right],
$$

is a symmetric tensor field of rank two. The aim is to recover the strain tensor field $\epsilon(x, y)$ from given values of its LRT.

\section{Finite element discretisation}

In this section, the finite element discretisation of the LRT is shown with triangular and quadrilateral meshes. Let $\left\{\phi_{n}\right\}_{n=1}^{N}$ be the set of all standard linear basis functions associated with the $\mathrm{N}$ vertices of the triangulation in the domain $\Omega$. The choice of basis functions for the forward problem is governed by considerations of computational cost and accuracy of the numerical model. This is often not an optimal choice for the inverse problem, where it may be more appropriate to adapt the basis expansion to physical limits of resolution of the reconstruction and to enforce equilibrium constraints in the admissible solutions. Tensor strain components are

$$
\begin{aligned}
& \epsilon_{11}(x, y)=\sum_{n} V_{1 n} \phi_{n}(x, y), \quad \epsilon_{22}(x, y)=\sum_{n} V_{3 n} \phi_{n}(x, y), \\
& \epsilon_{12}(x, y)=\sum_{n} V_{2 n} \phi_{n}(x, y)=\epsilon_{21},
\end{aligned}
$$

where $V_{k n}, k=1,2,3$, are unknowns coefficients to be determined. Hence, an equivalent formulation of equation (2) is

$$
\begin{aligned}
\mathrm{I}_{\epsilon}\left(x_{0}, y_{0}, \hat{\mathrm{n}}\right)= & \frac{1}{\ell} \int_{0}^{\ell}\left[\mathrm{n}_{1}\left(\sum_{n} \mathrm{~V}_{1 \mathrm{n}} \phi_{\mathrm{n}}\left(\mathrm{x}_{0}+s n_{1}, y_{0}+s n_{2}\right)\right) \mathrm{n}_{1}\right. \\
& +2 n_{1}\left(\sum_{n} \mathrm{v}_{2 n} \phi_{n}\left(x_{0}+s n_{1}, y_{0}+s n_{2}\right)\right) n_{2} \\
& \left.+n_{2}\left(\sum_{n} v_{3 n} \phi_{n}\left(x_{0}+s n_{1}, y_{0}+s n_{2}\right)\right) n_{2}\right] d s .
\end{aligned}
$$


Figure 1: Beam with triangular mesh.

(a) Tri mesh nodes

(b) Initial mesh

(c) First refinement
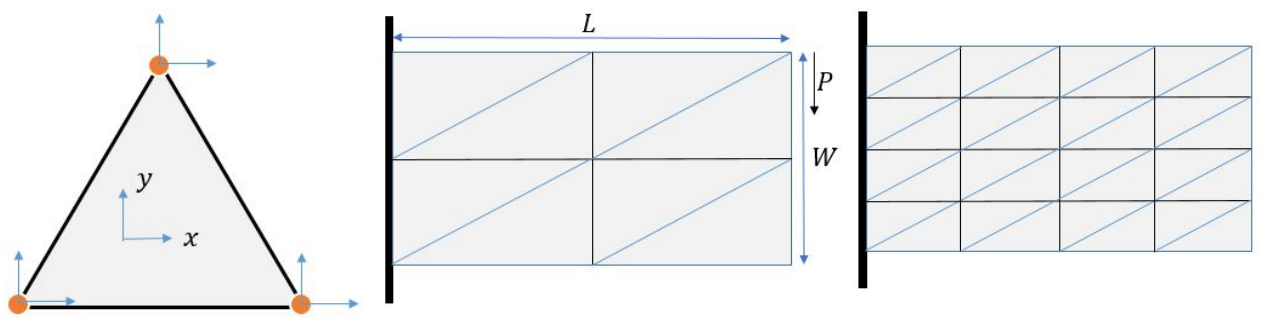

We write (5) in matrix notation:

$$
\mathrm{I}_{\epsilon}=\mathrm{K}_{\mathrm{ij}} \boldsymbol{\epsilon},
$$

where $I_{\epsilon}$ is the vector containing all of the Bragg edge strain field measurements, and $\boldsymbol{\epsilon}$ is the vector of order $3 \mathrm{~N} \times 1$ containing all unknown coefficients $V_{k n}$ for each element. Matrix $K_{i j}$ for $\{j=$ number of projections, $i=$ $3 \times N\}$ is formed from the evaluation of basis functions and the normal direction vector at each node of the triangulation shown in Figure 1a. The sum of the integral evaluation is then normalised by the path length. By evaluating the results from each node, the coefficient matrix $K_{i j}$ is formed. Note that for many projections taken, a maximum of 12 columns per row with non-zero elements corresponding to four intersecting nodes makes the $\mathrm{K}_{i j}$ matrix sparse. The system equation (6) is further reduced to a minimisation problem which can be solved by using least square fitting approach

$$
\min \left\|\mathrm{K} \boldsymbol{\epsilon}-\mathrm{I}_{\epsilon}\right\|_{2} \text {. }
$$

In practice, the number of unknown coefficients is relatively small compared to the amount of experimental data available. Hence, the system is usually over determined. Inverse problems are generally ill-posed, which means that a unique reconstruction of strain is not possible without imposing 
extra conditions $[8,1,6]$. Hence, to ensure the uniqueness of the solution, equilibrium equations are imposed implicitly and are defined as

$$
\begin{aligned}
& \frac{\partial}{\partial x}\left(\epsilon_{11}+v \epsilon_{22}\right)+\frac{\partial}{\partial y}(1-v) \epsilon_{12}=0 \\
& \frac{\partial}{\partial y}\left(\epsilon_{22}+v \epsilon_{11}\right)+\frac{\partial}{\partial x}(1-v) \epsilon_{12}=0
\end{aligned}
$$

where $v$ is Poisson's ratio. The equilibrium conditions are written in the weak formulation as follows:

$$
\begin{array}{ll}
\int_{\Omega}\left(\frac{\partial}{\partial x}\left(\epsilon_{11}+v \epsilon_{22}\right)+\frac{\partial}{\partial y}(1-v) \epsilon_{12}\right) \phi_{n}=0, & n=1, \ldots, N \\
\int_{\Omega}\left(\frac{\partial}{\partial y}\left(\epsilon_{22}+v \epsilon_{11}\right)+\frac{\partial}{\partial x}(1-v) \epsilon_{12}\right) \phi_{n}=0, & n=1, \ldots, N .
\end{array}
$$

Let

$$
\frac{\partial \epsilon_{l k}(x, y)}{\partial x}=N_{l k}, \quad \frac{\partial \epsilon_{l k}(x, y)}{\partial y}=M_{l k}, \quad l, k=\{1,2\},
$$

be the derivatives of the strain tensor field components. Now, since $\epsilon_{l k}$ are piecewise linear functions, their derivative with respect to $x$ and $y$, that is, $N_{l k}$ and $M_{l k}$, are piecewise constant. Hence, equation (9) is further written as:

$$
\begin{aligned}
& \sum_{T \in \mathcal{T}_{h}}\left[N_{11}+v N_{22}+(1-v) M_{12}\right] \int_{T} \phi_{n}=0, \\
& \sum_{T \in \mathcal{T}_{h}}\left[M_{22}+v M_{11}+(1-v) N_{12}\right] \int_{T} \phi_{n}=0,
\end{aligned}
$$

where $\mathcal{T}_{h}$ is the set of all triangles (or quadrilaterals) in the mesh. This gives another set of equations:

$$
\mathrm{C} \epsilon=0, \quad \mathrm{C}=\left[\begin{array}{l}
\mathrm{C}_{1} \\
\mathrm{C}_{2}
\end{array}\right]
$$

where $C_{1}$ and $C_{2}$ are the row vectors of equation (10). 
Figure 2: Beam with quadrilateral mesh.
(a) Quad mesh nodes
(b) Initial mesh
(c) First refinement
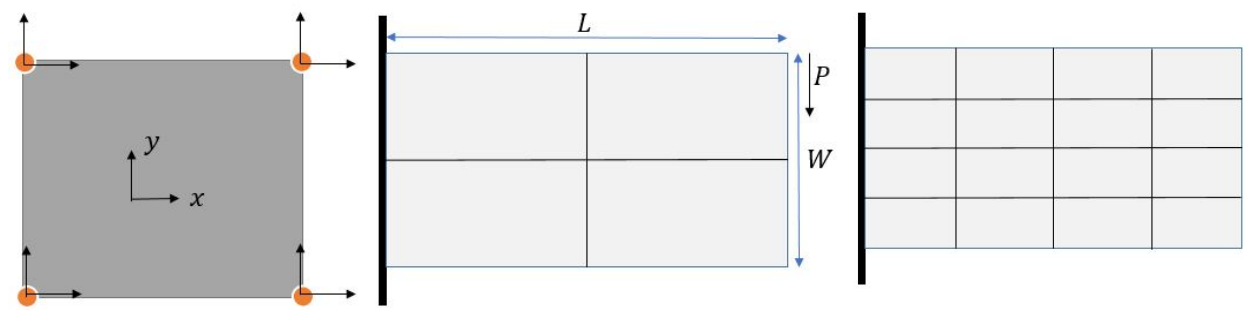

Similarly, if using quadrilateral elements, the discretized strain tensor field $\epsilon_{n}$ is expressed in terms of the standard bi-linear basis functions $\left\{\Psi_{n}\right\}_{\mathfrak{n}=1}^{N}$ associated with the $\mathrm{N}$ vertices of the quadrilateral mesh. The beam is then discretised into a structured mesh of non-overlapping quadrilateral elements, as seen in Figure 2.

In the quadrilateral mesh case $\epsilon_{i j}$ are piecewise bi-linear functions and their derivatives with respect to $x$ and $y$ are piecewise linear functions; that is, $N_{i j} \equiv N_{i j}(x, y)$ and $M_{i j} \equiv M_{i j}(x, y)$. Hence, the equilibrium equation (8) is integrated over each element $\mathrm{T}$, which leads us to

$$
\begin{aligned}
& \int_{\Omega}\left[N_{11}(x, y)+v N_{22}(x, y)+(1-v) M_{12}(x, y)\right]=0 \\
& \int_{\Omega}\left[M_{22}(x, y)+v M_{11}(x, y)+(1-v) N_{12}(x, y)\right]=0 .
\end{aligned}
$$

This provides another system of equations

$$
\mathrm{C} \epsilon=0,
$$

where $\mathbf{C}$ is the equilibrium integral matrix, which again has two rows. The problem is reduced to the following minimisation problem: find a vector $\boldsymbol{\epsilon}$ such that

$$
\min _{\mathbf{C} \epsilon=0}\left\|\mathbf{K} \epsilon-\mathrm{I}_{\epsilon}\right\|_{2} \text {. }
$$

The minimisation problem (14) is solved using a least squares method [9]. 


\section{Simulation results for a test case}

For analysis of strain parameters, a uniform isotropic cantilevered beam (plane stress) subject to a load $\mathrm{P}$, as shown in Figure 1b, is used as a test case. The strain values of a reference sample were obtained from the literature [5, 3, 2]. The domain is $\Omega=[0,12] \times[0,10]$ and the strain field within this plate is approximated by the analytic solution

$$
\epsilon(x, y)=\left[\begin{array}{cc}
(L-x) y & -\frac{(1+v)}{2}\left[\left(\frac{w}{2}\right)^{2}-y^{2}\right] \\
-\frac{(1+v)}{2}\left[\left(\frac{w}{2}\right)^{2}-y^{2}\right] & -v(L-x) y
\end{array}\right] \frac{P}{E I},
$$

where Young's modulus $\mathrm{E}=200 \mathrm{GPa}$, edge load $\mathrm{P}=2 \mathrm{kN}$, beam length $\mathrm{L}=12 \mathrm{~mm}$, beam height $\mathrm{W}=10 \mathrm{~mm}$, beam thickness $\mathrm{t}=3 \mathrm{~mm}$, poisson's ratio $v=0.3$.

The beam geometry was discretised into a number of elements using triangles and quadrilaterals as shown in Figures 1 and 2, respectively. The minimisation problem (14) was solved to obtain the reconstructed strain field. The $\mathrm{L}_{2}$ error was calculated between the true and reconstructed strain field.

In the practical scanning procedure, the data is collected as a set of projections, each of which defines a number of parallel lines sharing the same projection angle $\theta$. For our simulated example, the data consist of nine projections evenly spaced in $\left[0,180^{\circ}\right]$. The plot of beam reconstruction $L_{2}$ error as a function of the number of elements is shown in Figures 3 and 4 . Since $\epsilon_{x x}$ and $\epsilon_{y y}$ belong to the finite element space for quadrilaterals, numerical approximations reproduce these functions even with a coarser mesh. However, it can be seen from Figure 4 that the error of $\epsilon_{x y}$ has quadratic convergence. We also obtain quadratic convergence in the triangular case but error values are bigger compared to the quadrilateral case. 
Figure 3: log-log plot of the $\mathrm{L}_{2}$ convergence with triangular mesh.

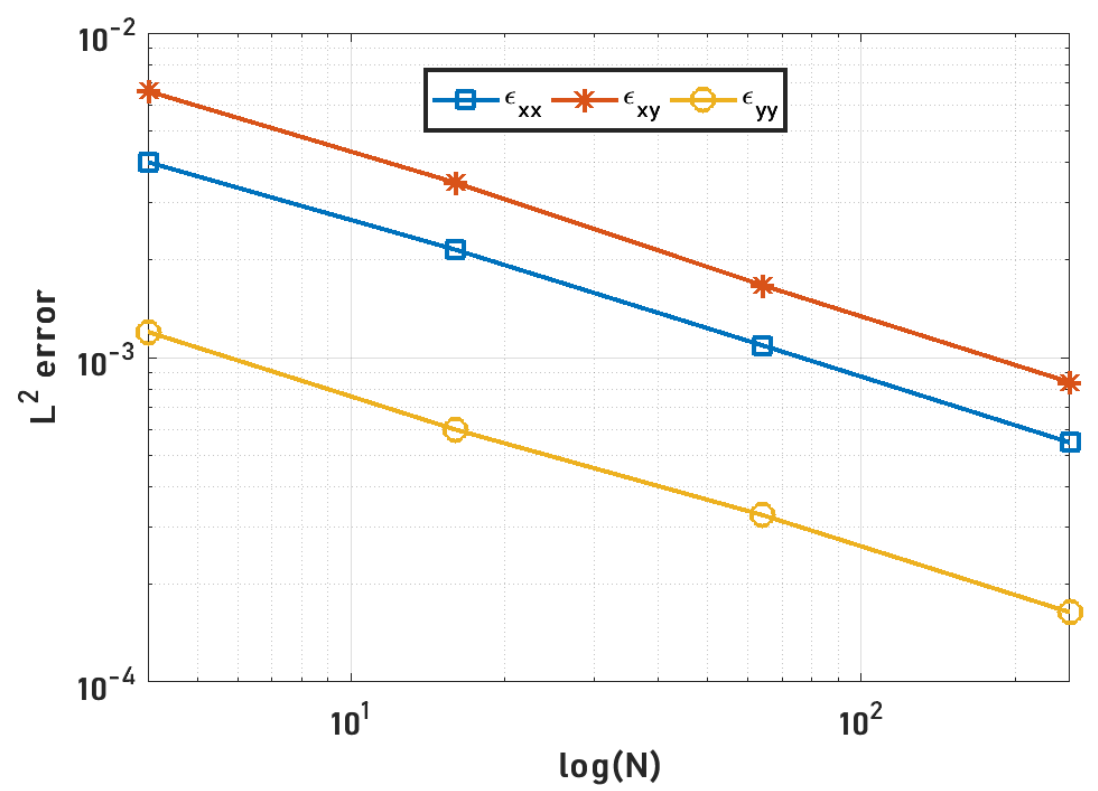

\section{Conclusion}

The reconstruction of the tensor strain field distribution in a cantilevered beam from a measurement of the transmission strain component has been demonstrated. The solution of the strain field was constructed by using standard basis functions, with a strain equilibrium condition enforced implicitly on the solution. We used a finite element method, with triangular and quadrilateral meshes to reconstruct the tensor strain field distribution in a cantilevered beam. The choice of basis functions for the forward mapping is governed by consideration of the computational cost and the accuracy of the numerical model. We hope that this work is an important step towards deciding which mesh should be considered for real-life problems. 
Figure 4: $\log$-log plot of the $\mathrm{L}_{2}$ convergence with quadrilateral mesh.

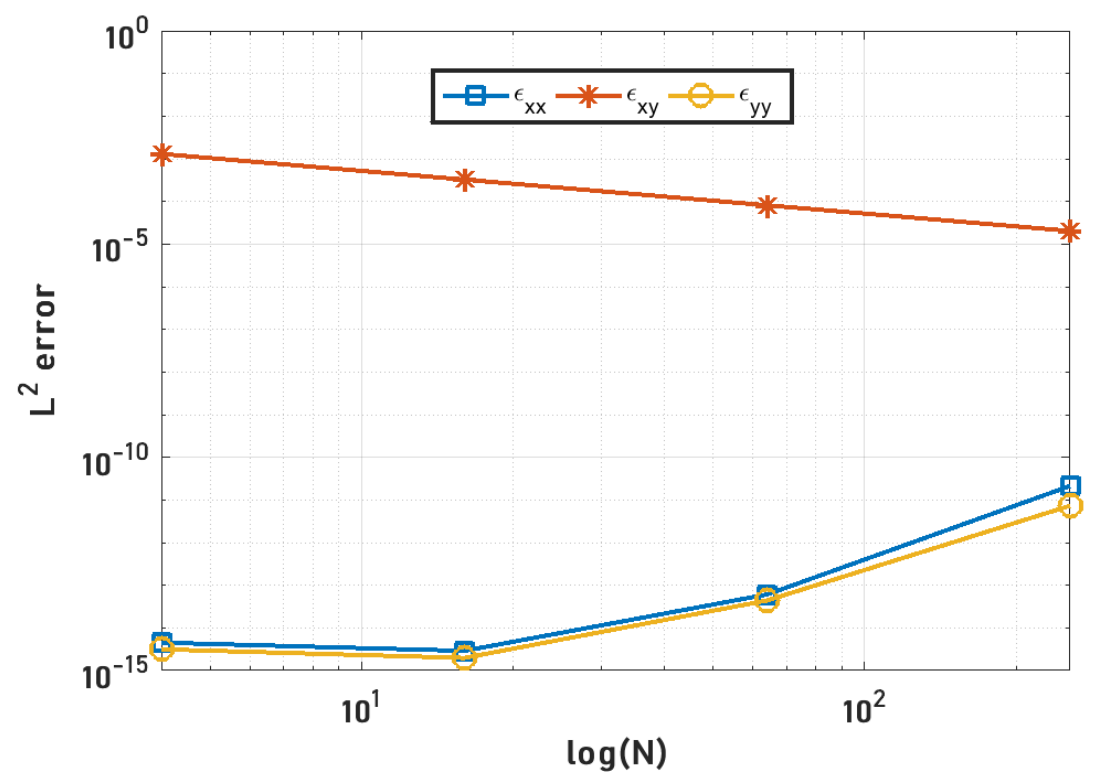

\section{Resources}

The Australian Research Council supports this work through a Discovery Project Grant. The scholarship for this project is supported by the ARC grant, UNIPRS, UNSR5050 Central School.

\section{References}

[1] B. Abbey, S. Y. Zhang, W. J. J. Vorster, and A. M. Korsunsky. "Feasibility study of neutron strain tomography". In: Proc. Eng. 1 (2009), pp. 185-188. DOI: 10.1016/j.proeng.2009.06.043 (cit. on p. C247).

[2] R. Aggarwal, M. H. Meylan, B. P. Lamichhane, and C. M. Wensrich. "Energy resolved neutron imaging for strain reconstruction using the 
finite element method". In: J. Imag. 6.3 (2020), p. 13. DOI: 10.3390/jimaging6030013 (cit. on p. C249).

[3] R. Aggarwal, M. H. Meylan, C. M. Wensrich, and B. P. Lamichhane. "Finite element approach to Bragg edge neutron strain tomography". In: Proceedings of the 18th Biennial Computational Techniques and Applications Conference, CTAC-2018. Ed. by B. Lamichhane, T. Tran, and J. Bunder. Vol. 60. ANZIAM J. June 2020, pp. C279-C294. DOI: 10.21914/anziamj.v60i0.14054 (cit. on p. C249).

[4] M. E. Fitzpatrick and A. Lodini. Analysis of residual stress by diffraction using neutron and synchrotron radiation. CRC Press, 2003. URL: https://www.routledge.com/Analysis-of-Residual-Stressby-Diffraction-using-Neutron-and-Synchrotron/FitzpatrickLodini/p/book/9780367446802 (cit. on p. C243).

[5] A. W. T. Gregg, J. N. Hendriks, C. M. Wensrich, A. Wills, A. S. Tremsin, V. Luzin, T. Shinohara, O. Kirstein, M. H. Meylan, and E. H. Kisi. "Tomographic reconstruction of two-dimensional residual strain fields from Bragg-edge neutron imaging". In: Phys. Rev. Appl. 10 (2018), p. 064034. DOI: 10.1103/PhysRevApplied.10.064034 (cit. on p. C249).

[6] J. N. Hendriks, A. W. T. Gregg, C. M. Wensrich, A. S. Tremsin, T. Shinohara, M. Meylan, E. H. Kisi, V. Luzin, and O. Kirsten. "Bragg-edge elastic strain tomography for in situ systems from energy-resolved neutron transmission imaging". In: Phys. Rev. Mat. 1 (2017), p. 053802. DOI: 10.1103/PhysRevMaterials.1.053802 (cit. on p. C247).

[7] E. H. Kisi and C. J. Howard. Applications of neutron powder diffraction. Vol. 15. Neutron Scattering in Condensed Matter. Oxford University Press, 2012. URL: https://global. oup.com/academic/product/applications-ofneutron-powder-diffraction-9780199657421 (cit. on p. C243). 
[8] W. R. B. Lionheart and P. J. Withers. "Diffraction tomography of strain". In: Inv. Prob. 31 (2015), p. 045005. DOI: 10.1088/0266-5611/31/4/045005 (cit. on p. C247).

[9] C. C. Paige and M. A. Saunders. "LSQR: An algorithm for sparse linear equations and sparse least squares". In: ACM Trans. Math. Software 8 (1982), pp. 43-71. DOI: 10.1145/355984.355989 (cit. on p. C248).

[10] J. R. Santisteban, L. Edwards, M. E. Fitzpatrick, A. Steuwer,

P. J. Withers, M. R. Daymond, M. W. Johnson, N. Rhodes, and

E. M. Schooneveld. "Strain imaging by Bragg edge neutron

transmission". In: Nucl. Inst. Meth. Phys. Res. 481 (2002), pp. 765-768. DOI: 10.1016/S0168-9002(01)01256-6 (cit. on pp. C243, C244).

[11] T. Shinohara and T. Kai. "Commissioning start of energy-resolved neutron imaging system, RADEN in J-PARC". In: Neut. News 26.2 (2015), pp. 11-14. DOI: 10.1080/10448632.2015.1028271 (cit. on p. C243).

[12] T. Shinohara, T. Kai, K. Oikawa, M. Segawa, M. Harada, T. Nakatani, M. Ooi, K. Aizawa, H. Sato, T. Kamiyama, H. Yokota, T. Sera, K. Mochiki, and Y. Kiyanagi. "Final design of the energy-resolved neutron imaging system RADEN at J-PARC". In: J. Phys. 746 (2016). DOI: $10.1088 / 1742-6596 / 746 / 1 / 012007$ (cit. on p. C243).

[13] A. S. Tremsin, J. B. McPhate, W. Kockelmann, J. V. Vallerga, O. H. W. Siegmund, and W. B. Feller. "High resolution Bragg edge transmission spectroscopy at pulsed neutron sources: proof of principle experiments with a neutron counting MCP detector". In: Nucl. Inst. Meth. Phys. Res. 633 (2011), S235-S238. DOI: 10.1016/j.nima.2010.06.176 (cit. on p. C243).

[14] R. Woracek, J. Santisteban, A. Fedrigo, and M. Strobl. "Diffraction in neutron imaging - A review". In: Nucl. Inst. Meth. Phys. Res. 878 (2018), pp. 141-158. DOI: 10.1016/j.nima.2017.07.040 (cit. on pp. C243, C244). 


\section{Author addresses}

1. R. Aggarwal, School of Mathematics and Physical Sciences, The University of Newcastle, AUSTRALIA. mailto:riya.aggarwal@uon.edu.au orcid:0000-0003-4261-8223

2. B. P. Lamichhane, School of Mathematics and Physical Sciences, The University of Newcastle, Australia. mailto:bishnu.lamichhane@newcastle.edu.au

3. M. H. Meylan, School of Mathematics and Physical Sciences, The University of Newcastle, Australia. mailto:mike.meylan@newcastle.edu.au

4. C. M. Wensrich, School of Engineering, The University of Newcastle, Australia.

mailto: christopher. wensrich@newcastle.edu.au 